\title{
Modelica Library for Building and Low-Voltage Electrical AC and DC Grid Modeling
}

\author{
Juan Van Roy ${ }^{1,2,3}$ Robbe Salenbien ${ }^{2,3}$ Johan Driesen ${ }^{1,3}$ \\ ${ }^{1}$ University of Leuven, Department of Electrical Engineering (ESAT) \\ Kasteelpark Arenberg 10, PB 2445, 3001 Leuven, Belgium \\ ${ }^{2}$ Flemish Institution for Technological Research (VITO) \\ Unit Energy Technology, 2600 Mol, Belgium \\ ${ }^{3}$ EnergyVille, 3600 Genk, Belgium
}

\begin{abstract}
This paper presents a Modelica library for electrical grid systems for low-voltage distribution grids and inbuilding grids. The library is based on previous work, in which a library was presented to simulate fully balanced three-phase low-voltage distribution grids [1]. This library is extended to simulate three-phase unbalanced low-voltage distribution grids as part of the IDEAS library [2]. The library also allows to simulate in-building $\mathrm{AC}$ and $\mathrm{DC}$ grids. The AC grids can be single-phase or three-phase (un)balanced grids. Electrical grids may connect many different energy systems (loads and generation units), different grids and/or buildings within districts. The library allows to assess the grid impact of these systems on different grid types. Control or optimization strategies can use grid variables, such as voltages and power exchanges.

Keywords: Electrical grid; AC grid; DC grid; Power flow analysis; Modelica
\end{abstract}

\section{Introduction}

Climate and energy goals are set, i.e. the European 20/20/20 targets [3]. One of these targets is an improvement in the EU's energy efficiency and the integration of renewable energy resources (RES) in the power production. Also, energy goals and benchmarks at the level of individual buildings are stated in the $\mathrm{Eu}-$ ropean Directive 2010/31/EU [4]. It is stated that by 2020 all new buildings need to be nearly zero-energy buildings (nZEB). nZEBs target a high penetration of RES, such as photovoltaic (PV) systems, and a high energy efficiency in the built environment.

This integration of RES and energy-efficient technologies in buildings may result in an increased elec- trification. Also different domains, such as the electrical and thermal domain, tend to become more integrated. This requires new approaches to analyze these integrated systems [5], such as taking into account the limitations of an electrical grid or using grid variables as control inputs in building design simulations. This may lead to a more effective analysis and better control of the energy system under consideration.

\subsection{Electricity grids}

\subsubsection{Radial versus meshed grids}

In the electrical power system, two types of electricity grids exist, namely distribution and transmission grids [6]. Distribution grids (low to medium voltage level) often differ fundamentally from transmission grids (high voltage level). Transmission grids are mostly meshed grids, whereas distribution grids are mostly radially. This means there is only one point of common coupling (PCC), which reduces the reliability of the distribution network. In case of a fault, all loads behind the fault will be switched off. Second, the R/X (resistance/reactance) ratio increases when the voltage level decreases. Thus, low voltage residential distribution grids are highly resistive.

Electricity grids in buildings are similar to lowvoltage distribution grids. As buildings are connected to the grid at one point, these grids are also radial. Loads (or buildings) can be single-phase or threephase connected to the grid. Electrical grids in buildings can be a combination of different single and threephase cables which connect the loads (see Fig. 1).

\subsubsection{AC versus DC in-building grids}

The current $\mathrm{AC}$ distribution grid and $\mathrm{AC}$ in-building grids are traditionally designed to feed $\mathrm{AC}$ and DC 

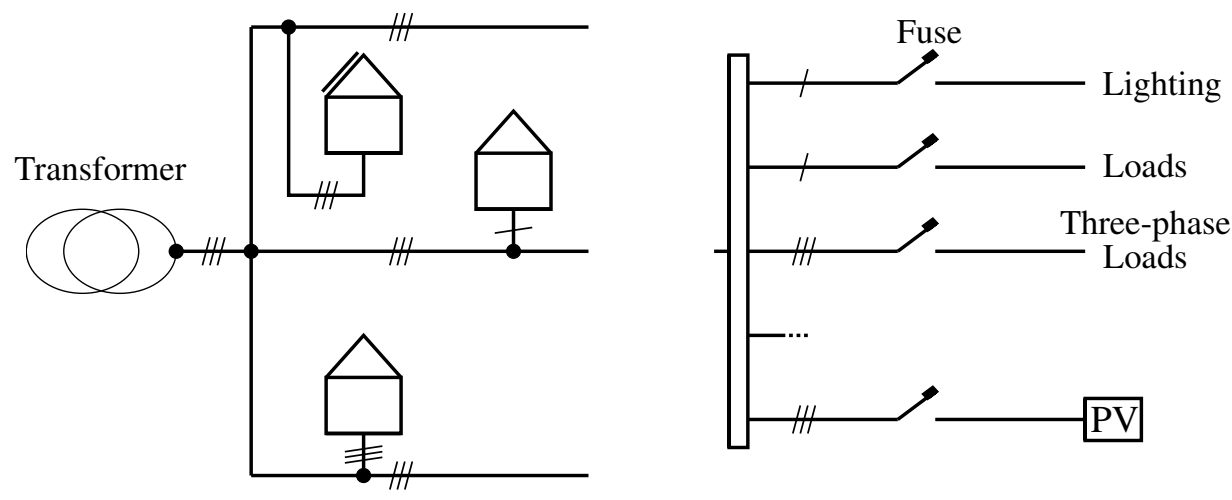

Figure 1: Comparison of low-voltage distribution grids (left) and in-building grids (right).

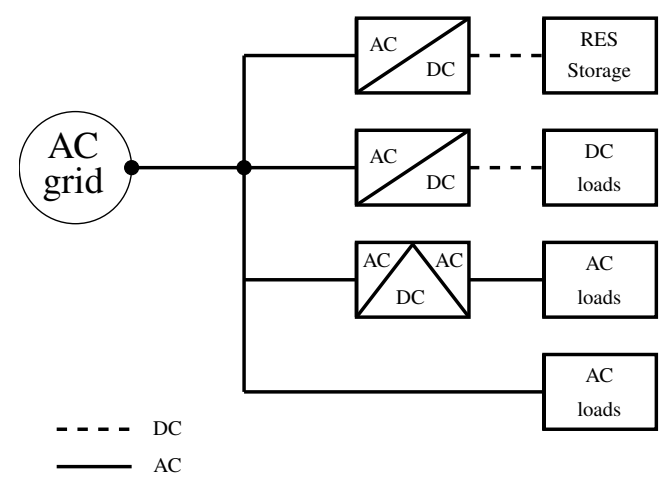

Figure 2: A traditional AC in-building grid with distributed converters (e.g. in appliances).

loads (e.g. household appliances) with distributed converters, e.g. rectifier in an appliance (see Fig. 2). Since almost all electric loads are equipped with power electronic converters, the interest in DC grids increases. It may lead to efficiency and economic advantages by reducing the losses [7]. Also, the harmonic distortion can be significantly reduced, which saves energy and improves the lifetime of equipment [8]. And a central AC/DC connection with the distribution grid can help to solve the three-phase balancing problem. Fig. 3 shows a possible lay-out of a hybrid AC/DC electrical grid for buildings. This grid includes e.g. a central $\mathrm{AC} / \mathrm{DC}$ converter, a central DC/DC converter for the DER, storage units, EVs, etc. and DC/DC converters for loads on different DC voltages.

\subsection{Electricity grid impact}

Energy efficiency can be achieved by a further electrification through new, more efficient technologies such as heat pumps and electric vehicles (EVs) [9]. However, from the electricity grid point of view, these new

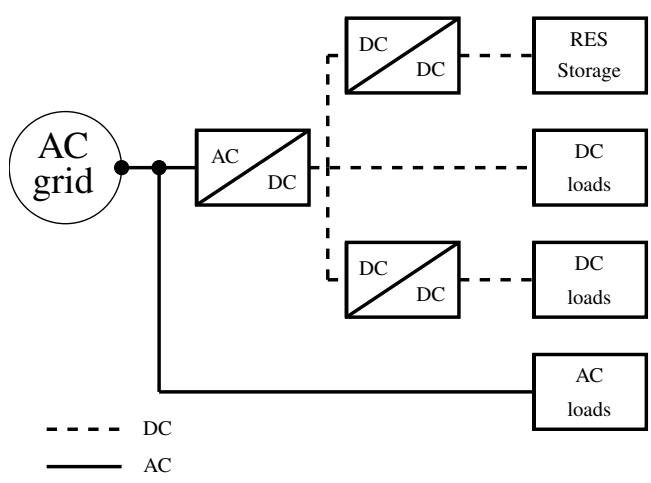

Figure 3: A possible hybrid AC-DC grid with a central bidirectional converter (AC/DC).

technologies have a twofold grid impact.

First, the power consumption will increase. For instance, when full EVs are charged only at home, the additional power consumption of about 2,350 to $3,750 \mathrm{kWh}$ doubles the average Flemish household's power consumption. On the other hand, local RES will introduce bidirectional power flows due to a certain non-simultaneity with the local demand [10].

Second, the increased power consumption and the intermittent production character of RES and its potential non-simultaneity with the local power demand have an impact on the low-voltage electricity grid [10]: both the distribution and in-building grid. The injection of electricity and the increased power consumption may lead to peak loads and higher resistive losses. As LV grids are mainly resistive, voltage deviations and phase unbalance occur due to the active power flows $[2,10,11]$. To minimize the grid impact, a proper synchronization of consumption and production of electricity and heat is needed through demand side management (DSM), electrical and thermal sto- 
rage and minimizing the power consumption [9].

Taking into account the limitations of an electrical grid or using grid variables as control inputs in building design simulations, may lead to a more effective analysis and better control of the energy system under consideration.

\subsection{Scope of paper}

To investigate the impact of energy systems on the electrical distribution grid or in-building grid, a library is developed to simulate both single and threephase unbalanced AC radial grids and DC electrical grids. For AC grid analyses, a quasi-stationary model is implemented, assuming the frequency is fixed (e.g. $50 \mathrm{~Hz}$ ). This allows to represent the waveforms by its amplitude and phase shift. Therefore, dynamic transient are not included.

In previous work, the first version of this library was presented [1]. This library consisted of models for the modeling of balanced three-phase low-voltage distribution grids, which can be represented by an equivalent single-phase grid. This paper discusses the recent additions: the unbalanced three-phase lowvoltage grids and DC grids.

First, the basics on power flow analyses is given in Section 2. Section 3 and 4 describe the physical and the model description in Modelica. To conclude, a comparative model validation is performed.

\section{Power flow analysis}

A power flow analysis is performed to obtain the voltage and current information in each node and line of the electrical grid, based on the Laws of Kirchhoff:

Conservation of electric charge The sum of currents flowing into a node is equal to the sum of currents flowing out of a node.

Conservation of energy The sum of the voltage drops in any closed circuit is zero.

The voltage drop $\Delta v$ in a line $k$ between nodes $n$ and $n+1$ is defined as:

$$
\Delta v_{k}(t)=v^{n}(t)-v^{n+1}(t)=Z_{k} i_{k}(t),
$$

with $Z_{k}$ the impedance of the line and $i_{k}$ the line current. When the nodal currents, line currents and nodal voltages are known, the apparent power in one phase $S_{f}$ can be calculated. $S$ consists of active power $P$ and reactive power $Q$ :

$$
S_{f}(t)=P_{f}(t)+j Q_{f}(t)=v_{f} i_{f}^{*},
$$

with a non-linear relation between $S_{f}, v_{f}$ the phase voltage and $i_{f}^{*}$, the complex conjugate of the total phase current $i_{f}$. The total apparent power is calculated as $S(t)=\sum S_{f}$. For DC grids: $Q=0$.

The joule losses $P_{J}$ in a grid are the sum of the losses in all phases and neutral (or negative) conductor. The joule losses in a line $k$ are calculated as follows:

$$
P_{J, k}=R_{k}\left|i_{k}\right|^{2},
$$

with $R_{k}$ the resistance of a line $k$. Note that also the reactive current is responsible for a part of $P_{J}$.

The non-linear system requires numerical methods to obtain a solution. Several methods are available to solve a power flow analysis, such as direct and iterative methods. The backward-forward sweep is an example of an iterative method, which is well suited for radial grids. This method is illustrated in Appendix A. In Dymola, the DASSL solver [12] is used to solve the power flow analysis.

In [13], a three-phase unbalanced power flow analysis is implemented in MATLAB. This model uses the backward-forward sweep technique. The models in the developed Modelica library will be validated with this MATLAB model. Other available Modelica libraries regarding electrical systems are e.g. the SPOT library [14] and Electric Power Library [15] which allow both steady-state and transient simulations.

The library in this paper allows to do a quasistationary analysis of electrical grids. This allows to represent the waveforms by its amplitude and phase shift. Dynamic transient are not included.

\section{Physical model description}

\subsection{Grid topology representation}

Traditionally, radial grids are represented by an incidence matrix (or connection matrix) T. Eq. (4) gives an example of an incidence matrix of a grid in which consecutive nodes are connected.

$$
\mathbf{T}=\left[\begin{array}{cccccc}
-1 & 0 & 0 & \cdots & 0 & 0 \\
1 & -1 & 0 & \cdots & 0 & 0 \\
0 & 1 & -1 & \cdots & 0 & 0 \\
\vdots & \vdots & \vdots & \ddots & \vdots & \vdots \\
0 & 0 & 0 & \cdots & -1 & 0 \\
0 & 0 & 0 & \cdots & 1 & -1
\end{array}\right] .
$$

The columns correspond with the number of nodes (or connection points), whereas each row is a segment of the grid (line) between two nodes. The start and end node of each line are represented by respectively 
1 and -1 . Since a radial grid contains $n$ nodes, there are $(n-1)$ line segments. To attain a square matrix, an additional (first) row is introduced to represent the imaginary line segment between the transformer and the first node (with a length of $0 \mathrm{~m}$ ). This line segment only has an end node.

\subsection{Grid elements}

\subsubsection{Cables}

Cables, with a length $f_{L}(\mathrm{~m})$, for the line segments are characterized by an impedance $Z=R+j X$, with $R$ the resistance and $X$ the reactance of the cable:

$$
R=f_{L} r \quad \text { and } \quad X=f_{L} x,
$$

with $r$ the characteristic resistance and $x$ the characteristic reactance in $\Omega / \mathrm{m}$. This allows to represent the grid with an impedance matrix $\mathbf{Z}=\mathbf{R}+j \mathbf{X}$. Single-phase AC and DC grids contain two conductors, respectively a phase/neutral conductor and a positive/negative (or positive/neutral) conductor. Threephase grids have three phases and one neutral conductor (total of four conductors).

For three-phase AC cables, typically nominal $\Pi$ or $T$ models are used to model cable, which include the resistance and reactance of the cable, as well as the shunt admittance. This capacitance is neglected for the considered low-voltage grids in this paper. Also, it is assumed that all phases are symmetrically spaced and that all phases are regularly transposed. Compared to [13], the shunt admittance and mutual impedance, which has a very small impact for low-voltage cables, is neglected.

\subsubsection{Transformers}

A transformer transforms e.g. a higher to a lower AC three-phase voltage level. The transformer is modeled with a phase impedance $Z_{\mathrm{tr}}=R_{\mathrm{tr}}+j X_{\mathrm{tr}}$, which are assumed identical for the three phases. The losses in the transformer $P_{\text {loss }}$ are the sum of the no-load losses $P_{0}$, which are assumed to be constant, and the sum of the joule losses $P_{J}$ in each phase $f$ of the transformer:

$$
P_{\text {loss }}=P_{0}+P_{J}=P_{0}+\sum_{f=1}^{3} R_{\mathrm{tr}}\left|i_{f, k}\right|^{2} .
$$

\subsection{Load models}

\subsubsection{Loads and generation units}

Loads and generation units are modeled as constant power loads, which is common for loads equipped with power electronic. This means the power is not depending on the solution of the power flow (voltage and current). This results in a non-linear relation between power, voltage and current, as shown in Eq. (2) and its implementation in Section 4.2.4. Other possible load models take e.g. grid variables as input, such as voltage-droop load models [16].

\subsubsection{Converters}

Converters are required to convert $\mathrm{AC}$ to $\mathrm{DC}$ (rectifiers) or vice versa (inverters) or DC to DC. For rectifiers, the DC power $\left(P_{\mathrm{DC}}\right)$ is lower (or equal) than the AC power $\left(P_{\mathrm{AC}}\right)$. For inverters: $P_{\mathrm{AC}} \leq P_{\mathrm{DC}}$. The ratio between the DC and AC power is defined by the efficiency of the converter. The power electronics level of converters is not modeled.

Converters can also regulate the reactive power consumption or injection [13]. The power factor (pf $=P /|S|$ ) can be leading (drawing reactive current) or lagging (injecting reactive current).

\section{Implementation in Modelica}

This section gives an overview on the functional requirements of the models, which are identified from the different use cases. In the second part, the component modeling is described.

\subsection{Requirements}

\subsubsection{Use cases}

This section describes the needs for electrical grid modeling at the level of an individual building and at the level of a district energy system. The following use cases are identified, which are used to define the functional requirements:

- Grid impact analyses of electrical processes at building and district level. The results are an analysis of the nodal voltages, line currents, powers (active and reactive) and power losses.

- Grid architecture: single/three-phase AC grids and DC grids.

- Flexible and scalable approach for grid topology definition for all grid types.

- Connection architecture: Single/three-phase connection for loads and generation units.

- Integrated control or optimization by using grid variables, such as the nodal voltages, powers, etc. 


\subsubsection{Functional requirements}

This section describes the functional requirements. Physics to be modeled include:

- Load and generation units:

- Active and reactive power;

- Connectors: Complex voltage/current and single/three-phase;

- Converter losses.

- Electrical grids:

- Quasi-stationary analysis;

- Active and reactive power flows and losses;

- Transformer: voltage drops and losses;

- Unbalanced loads (including the resulting zero-point shifting);

- Grid type:

* Distribution and in-building grid;

* Single-phase, three-phase (unbalanced) and equivalent single-phase;

* AC and DC.

- Integrated control or optimization: access to grid variables.

\subsection{Modelica model implementation}

\subsubsection{Connectors}

The library uses the connectors from the Standard Modelica Library:

- AC connectors: Electrical.Quasistatio nary.SinglePhase. Interfaces.Pin

- DC connectors: Electrical. Analog. In terfaces.Pin

These connectors contain the voltage and current (flow). The AC positive and negative pin also include a reference angle.

The grid contains both internal and external nodes. The internal nodes include the neutral or negative connector, while the external nodes are used to connect the loads and generation units.

External nodes The external connection nodes are defined as: .Pin Nodes [numPha, nNodes], with nNodes the number of grid nodes and numPha=1 for single-phase AC and DC loads and generation units and numPha $=3$ for three-phase $\mathrm{AC}$ loads and generation units.
Internal nodes The internal connection nodes are defined as .Pin gridNodes[numCon, nNodes], with numCon the number of conductors (see Section 3.2).

Adapters Adapters are available to connect the internal and external nodes (see Code 1). For singlephase and DC grids, the adapter connects the two wire to a single wire system. For three-phase grids these are respectively four and three wire systems. A singlephase load or generation unit can be connected to one phase of a three-phase grid.

Fig. 4 shows the use of an adapter in a three-phase grid to connect the internal (node $4 \mathrm{Lines}$ ) and external nodes (nodes $3 \mathrm{Ph}$ ). The electricity grid connects the gridConnection (e.g. voltage source or transformer) with the internal nodes.

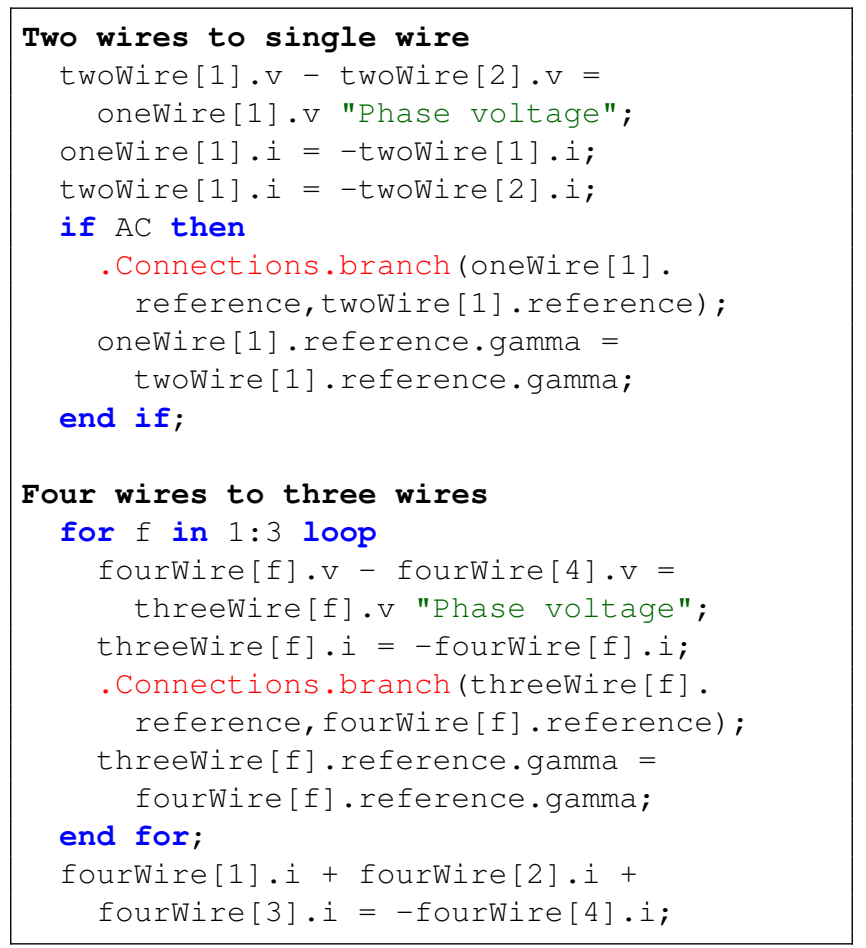

Code 1: Adapters for internal and external nodes.

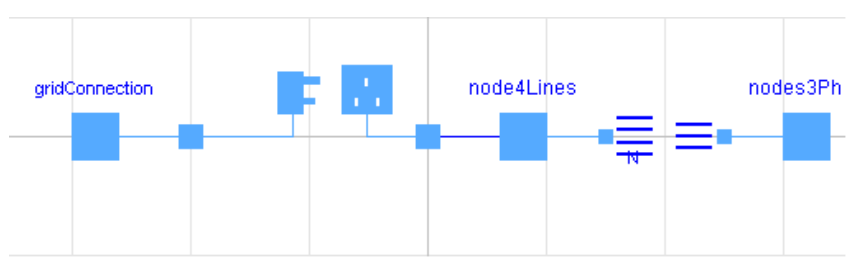

Figure 4: Use of adapter for three-phase grids. 


\subsubsection{Grid topology}

The grid topology is described by the incidence matrix and the cable impedances (see Section 3). This is shown in Code 2. GridType extends GridImp and describes the grid topology.

Code 3 shows how the grid is constructed by using the incidence matrix. This code is used to construct each phase (and neutral) of the grid by connecting the different line segments (conductor) and grid nodes.

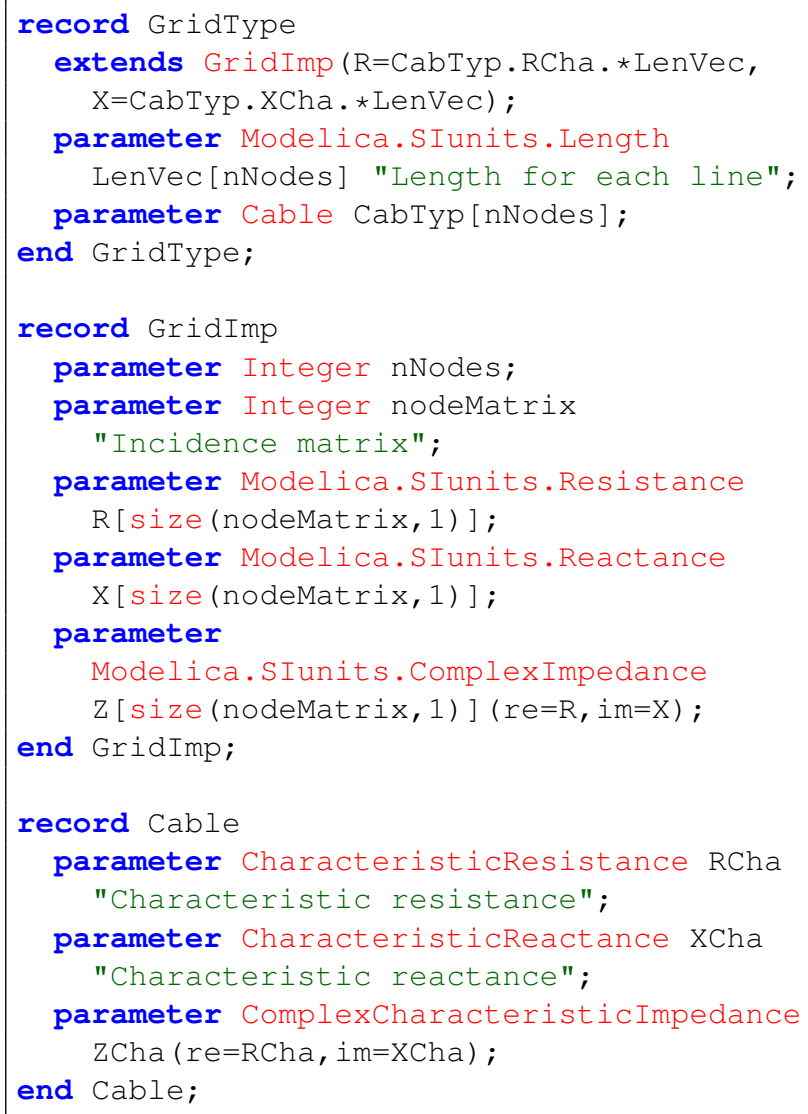

Code 2: Description of grid topology (interfaces).

\subsubsection{Grid elements}

Cables Code 2 also describes the record Cable. This record describes each cable type with their respective characteristic impedance, which is the impedance per unit of length $(\Omega / \mathrm{m})$.

Transformers The transformer model consists of an impedance for each of the three phases. The interface for the records, which are used to define the transformer data, is shown in Code 4.

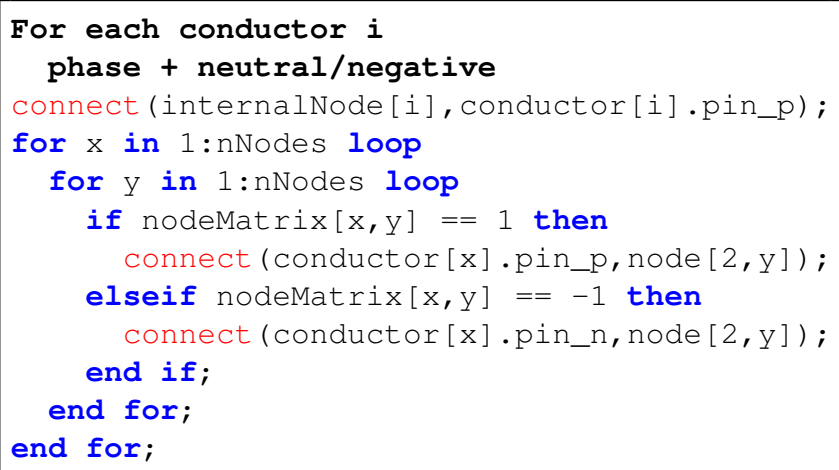

Code 3: Connect statements for the grid construction.

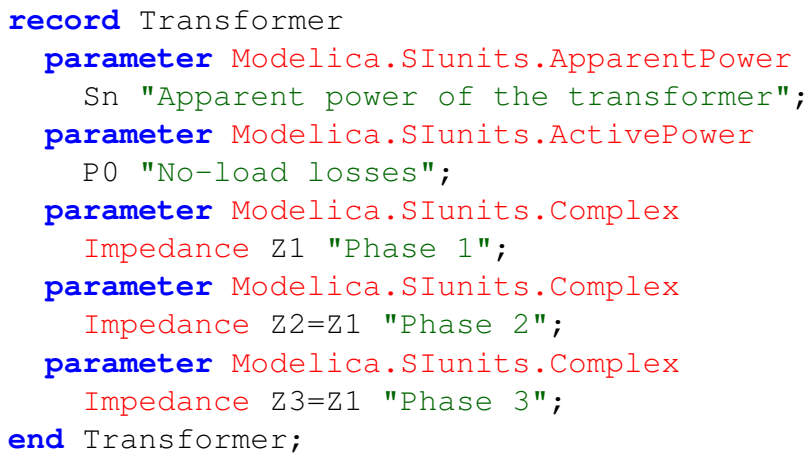

Code 4: Transformer description (interface).

\subsubsection{Load models}

Constant power model Code 5 shows the nonlinear relation between the apparent power, voltage and current shown in Eq. (2).

Converters The converters are implemented according to Section 3.3.2 and the power equations in Section 2. Fig. 5 shows the diagram for a bidirectional converter, which uses the AC and DC connectors as defined in Section 4.2.1. The AC connector can be single or three-phase. For three-phase converters, the apparent power is equally divided over the three phases: $S_{f}(t)=S(t) / 3$.

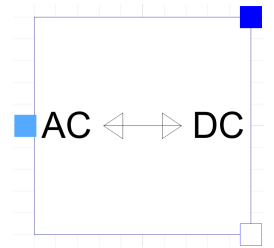

Figure 5: Use of adapter for three-phase grids. 


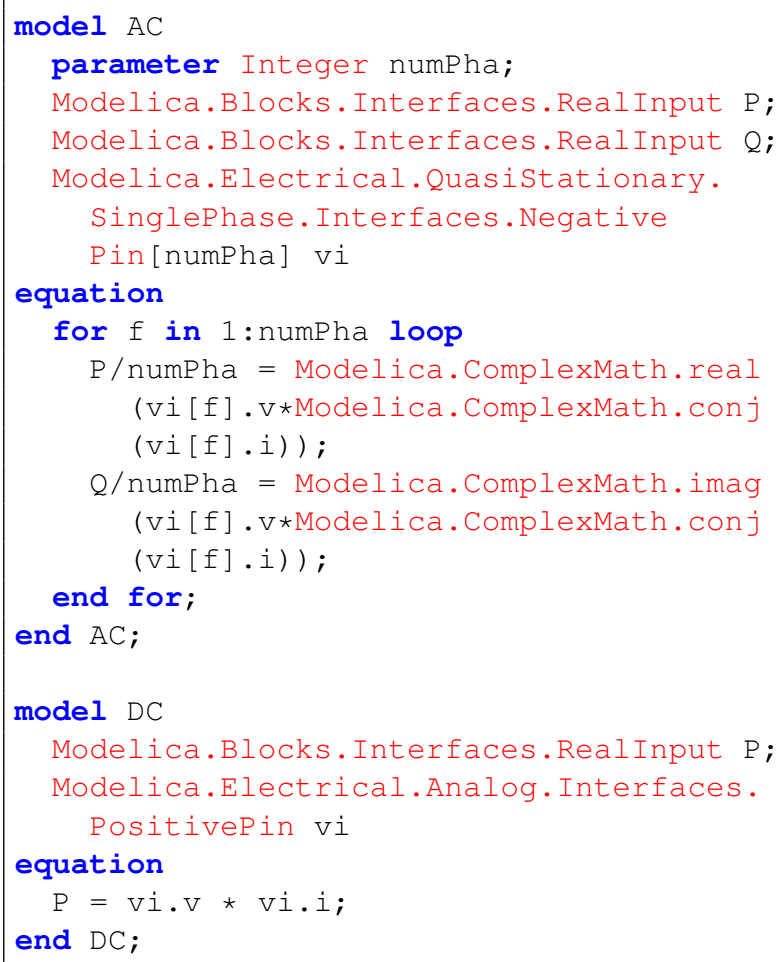

Code 5: Relation apparent power, voltage and current for both AC (single/three-phase) and DC systems.

Code 6 shows the implementation of a bidirectional converter which extends the partial Converter model. A boolean is used to define the operation (rectifier or inverter) in function of the sign of the DC power. For the Rectifier and Inverter model, this boolean is a parameter.

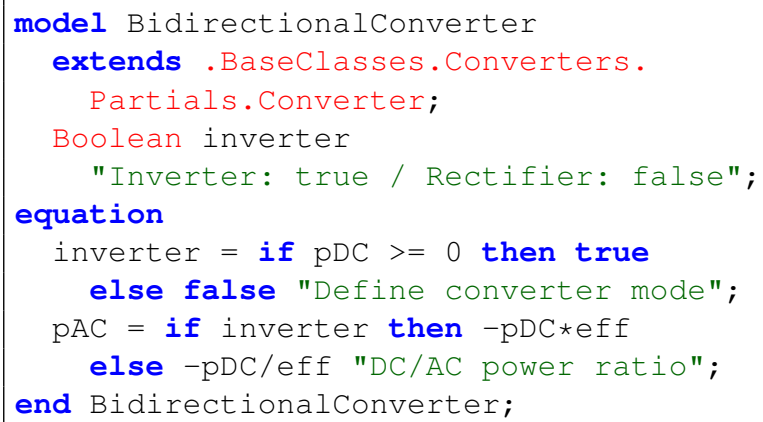

Code 6: Converter mode and DC/AC power ratio.

\subsection{Simulation of multiple grid types}

The Modelica implementation also allows to simulate multiple types of grid (i.e. single-phase AC, three- phase AC and DC grids) in one simulation. Fig. 6 shows an example with different grids (with loads) connected to one feeding transformer. For the DC grid, a converter is required as well as a separate DC grounding.

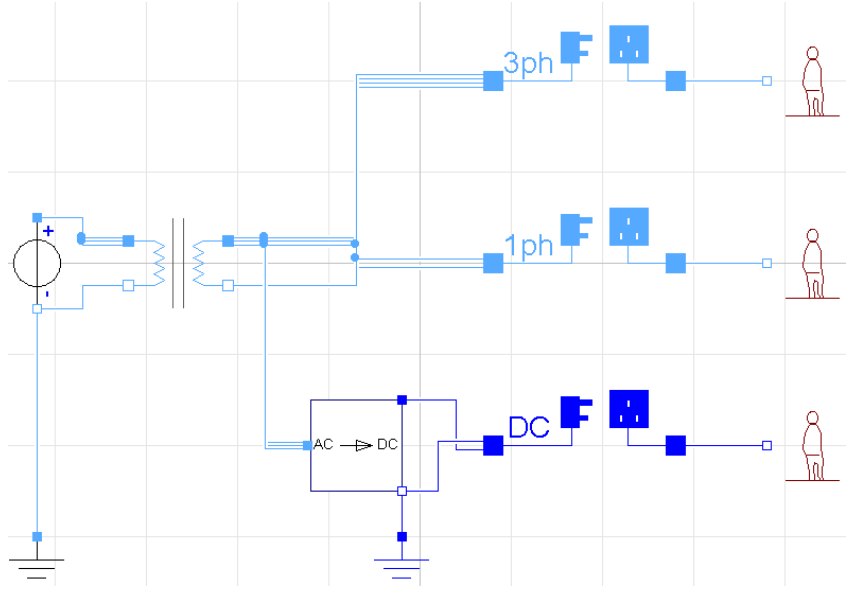

Figure 6: Simulation of multiple grid types in one simulation.

\section{Comparative model validation}

The electrical grid models are validated using a comparative validation method. The three-phase grid models from the Modelica library are compared with the power flow analysis tool in [13].

\subsection{Example case: residential district}

A simple case study is developed with a small threephase unbalanced residential distribution grid.

There is one feeder connected to the feeding transformer. 20 residential loads are connected to respectively one of the 20 nodes in this feeder. The cables between the nodes have a length of $16 \mathrm{~m}$ and have a characteristic impedance of $0.507+j 0.229 \mathrm{~m} \Omega / \mathrm{m}$. The feeder is connected to a three-phase transformer which has a phase impedance of $20.4+j 67.5 \mathrm{~m} \Omega$. The nominal phase voltage between a phase and the neutral conductor is $230 \mathrm{~V}$. The grid topology is illustrated in Fig. 7 (not on scale).

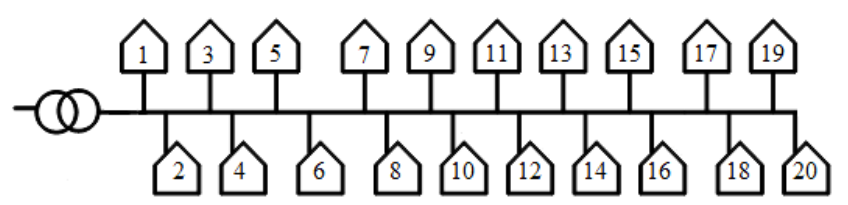

Figure 7: Grid topology for the validation model. 
Synthetic residential load profiles for one day are available on a 30-minute resolution [17]. A set of 16 different profiles are randomly distributed over the different household connections to the distribution grid. The household loads are all single-phase connected.

\subsection{Validation results}

For the comparative validation, the results on the nodal phase voltages are compared with the results of the models from [13]. Fig. 8 shows the absolute ${ }^{1}$ difference in results between the Modelica and Matlab models. Box plots are used to show the difference in the nodal phase voltages during the simulation period of one day for each phase of the electrical grid. The median, minimum and maximum values of these absolute differences are repeated in Table 1.

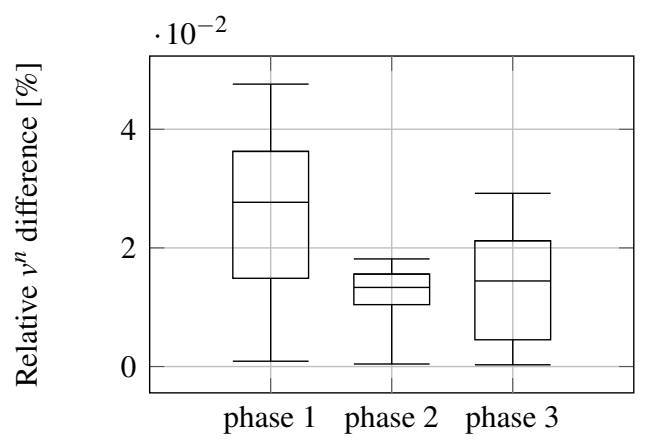

Figure 8: Relative absolute difference on the nodal phase voltages for each phase between the Modelica models and the Matlab models from [13].

Table 1: Median, average, minimum and maximum relative nodal voltage difference [\%] for each phase.

\begin{tabular}{lcccc}
\hline & Median & Average & Min. & Max. \\
\hline Phase 1 & 0.0277 & 0.0256 & 0.0009 & 0.0476 \\
Phase 2 & 0.0133 & 0.0121 & 0.0004 & 0.0181 \\
Phase 3 & 0.0144 & 0.0135 & 0.0003 & 0.0292 \\
\hline
\end{tabular}

The average absolute nodal phase voltage differences for a voltage of $230 \mathrm{~V}$ are in the order of $10^{-2} \mathrm{~V}$. The minimum difference is less than $1 \mathrm{mV}$. Note that in this case study the nodal phase voltages are always lower than $230 \mathrm{~V}$ since there are only loads.

This difference between both models is function of the loads and the grid topology, since an error in one node will propagate through the grid as a result of the Laws of Kirchhoff in an electrical circuit. Therefore,

\footnotetext{
${ }^{1}$ The differences are both positive and negative
}

the difference in results will also increase for nodes further from the feeding point. A second source of the difference in results is the stop criterion for the iteration in both simulations. The Matlab code uses a maximum allowed voltage error $(1 \mathrm{mV})$ as a stop criterion. In Dymola, a tolerance of $10^{-4}$ is used. A last part of the difference is that the shund admittance and mutual impedance between cables in three-phase systems is neglected in this Modelica library.

The differences between the results of both models are limited. Since both stop criteria for the iterative solution method is different, the results show that the accuracy is sufficient to apply this Modelica library.

\section{Conclusions}

A Modelica library is developed to simulate singlephase AC radial grids, both balanced and unbalanced three-phase AC radial grids and DC electrical grids. For AC grid analyses, a quasi-stationary model is implemented, assuming a fixed grid frequency (e.g. $50 \mathrm{~Hz}$ ). The library is based on previous work, in which a library was presented to simulate fully balanced three-phase low-voltage distribution grids [1].

The models in this library can be used for electrical grid systems for low-voltage distribution grids and in-building grids. Electrical grids may connect many different energy systems (loads and generation units), different grids and/or buildings within districts. The library allows to assess the grid impact of these systems. Control or optimization strategies can use grid variables, such as voltages and power exchanges.

The comparative validation of this Modelica library with a power flow simulation in Matlab [13] shows that the difference in nodal voltages depends on the loads, the grid topology and end criterion. Also the mutual impedance of cables in three-phase systems is neglected. Nevertheless, the average voltage differences are limited, for this case study in the order of $10^{-2} \mathrm{~V}$ for a voltage of $230 \mathrm{~V}$.

\section{A Appendix: Backward-forward sweep method}

The backward-forward sweep is an example of an iterative method to solve a power flow analysis. The method is well suited for radial grids.

First, an initial guess of the voltage profile is set. This allows the backward step to calculate the nodal $\left(\mathbf{I}_{\text {node }}\right)$ and line currents ( $\left.\mathbf{I}_{\text {line }}\right)$ in function of the ap- 
parent power $\left(\mathbf{S}_{\text {node }}\right)$, the nodal voltages $\left(\mathbf{U}_{\text {node }}\right)^{2}$ and incidence matrix (T), which is defined in Section 3.1:

$$
\begin{gathered}
\mathbf{I}_{\text {node }}=f\left(\mathbf{S}_{\text {node }}, \mathbf{U}_{\text {node }}\right)=\left(\frac{\mathbf{S}_{\text {node }}}{\mathbf{U}_{\text {node }}}\right)^{*}, \\
\mathbf{I}_{\text {line }}=(\operatorname{transpose}(\mathbf{T}))^{-1} \cdot \mathbf{I}_{\text {node }} .
\end{gathered}
$$

In the forward step, the nodal voltage is calculated with the line currents: $\mathbf{U}_{\text {node }}=\mathbf{U}_{\text {grid }}-\mathbf{Z} \cdot \mathbf{I}_{\text {line }}$. The iteration stops when convergence is reached.

\section{Acknowledgments}

The work of J. Van Roy is funded through a doctoral scholarship of the Flemish Institute for Technological Research and the KIC InnoEnergy PhD School.

\section{References}

[1] B. Verbruggen, J. Van Roy, R. De Coninck, R. Baetens, L. Helsen, and J. Driesen, "ObjectOriented Electrical Grid and Photovoltaic system modelling in Modelica," in 8th International Modelica Conference, Dresden, Germany, Mar. 2011.

[2] R. Baetens, R. De Coninck, J. Van Roy, B. Verbruggen, J. Driesen, L. Helsen, and D. Saelens, "Assessing Electrical Bottlenecks at Feeder Level for Residential Net Zero-Energy Buildings by Integrated System Simulation," Applied Energy, vol. 96, pp. 74-83, Aug. 2012.

[3] The European Commission. (2013, Dec.) The EU Climate and Energy Package. [Online]. Available: http://ec.europa.eu/clima/policies/package/

[4] The European Parliament, "Directive 2010/31/EU of the European Parliament and the Council on 19 May 2010 on the Energy Performance of Buildings (recast), 2010.

[5] J. Van Roy, B. Verbruggen, and J. Driesen, "Ideas for tomorrow: New tools for integrated building and district modeling," IEEE Power \& Energy Magazine, vol. 11, no. 5, pp. 75-81, Aug. 2013.

[6] H. Lee Willis, Power Distribution Planning Reference Book. USA: Marcel Dekker, Inc., 1997.

\footnotetext{
${ }^{2} \mathbf{I}_{\text {node }}, \mathbf{I}_{\text {line }}, \mathbf{S}_{\text {node }}$ and $\mathbf{U}_{\text {node }}$ are all complex matrices.
}

[7] P. Savege, R. Nordhaus, and S. Jamieson, "DC Microgrids: Benefits and Barriers." Yale School of Forestry \& Environmental Studies.

[8] B. Williamson, M. Redfern, R. Aggarwal, J. Allinson, C. Harris, P. Bowley, and R. Hotchkiss, "Project Edison: SMART-DC," in IEEE PES Innovative Smart Grids Technologies Europe (ISGT), Manchester, United Kingdom, Dec. 2011.

[9] International Energy Agency, "Energy Technology Perspectives 2012: Pathways to a Clean Energy System,” 2012.

[10] R. Passey, T. Spooner, I. MacGill, M. Watt, and K. Syngellakis, "The Potential Impacts of GridConnected Distributed Generation and How to Address Them: A Review of Technical and Non-Technical Factors," Energy Policy, vol. 39, no. 10, pp. 6280-6290, Oct. 2011.

[11] K. Clement, E. Haesen, and J. Driesen, "The Impact of Vehicle-to-Grid on the Distribution Grid," Electric Power System Research Journal, vol. 81, no. 1, pp. 185-192, Jan. 2011.

[12] L. Liu, F. Felgner, and G. Frey, "Comparison of 4 Numerical Solvers for Stiff and Hybrid Systems Simulation," in IEEE Conference on Emerging Technologies and Factory Automation, Bilbao, Spain, Sep. 2010.

[13] J. Tant, F. Geth, D. Six, and J. Driesen, "Multiobjective Battery Storage to Improve PV Integration in Residential Distribution Grids," IEEE Transaction on Sustainable Energy, vol. 4, no. 1, pp. 182-191, Jan. 2013.

[14] SPOT library. [Online]. Available: https://github. com/modelica-3rdparty/SPOT

[15] Modelon. Electric Power Library. [Online]. Available: http://www.modelon.com/products/ modelica-libraries/electric-power-library

[16] F. Geth, N. Leemput, J. Van Roy, J. Büscher, R. Ponnette, and J. Driesen, "Voltage Droop Charging of Electric Vehicles in a Residential Distribution Feeder," in IEEE PES Innovative Smart Grid Technologies (ISGT) Europe, Berlin, Germany, Oct. 2012.

[17] (2013, Dec.) Flemish Regulator for the Energy and Gas markets (VREG). [Online]. Available: http://www.vreg.be 